\title{
Analysis of a normalised expressed sequence tag (EST) library from a key pollinator, the bumblebee Bombus terrestris
}

\author{
Ben M Sadd ${ }^{1 *}$, Michael Kube ${ }^{2}$, Sven Klages ${ }^{2}$, Richard Reinhardt ${ }^{2}$, Paul Schmid-Hempel ${ }^{1}$
}

\begin{abstract}
Background: The bumblebee, Bombus terrestris (Order Hymenoptera), is of widespread importance. This species is extensively used for commercial pollination in Europe, and along with other Bombus spp. is a key member of natural pollinator assemblages. Furthermore, the species is studied in a wide variety of biological fields. The objective of this project was to create a $B$. terrestris EST resource that will prove to be valuable in obtaining a deeper understanding of this significant social insect.

Results: A normalised cDNA library was constructed from the thorax and abdomen of $B$. terrestris workers in order to enhance the discovery of rare genes. A total of $29^{\prime} 428$ ESTs were sequenced. Subsequent clustering resulted in 13 '333 unique sequences. Of these, 58.8 percent had significant similarities to known proteins, with 54.5 percent having a "best-hit" to existing Hymenoptera sequences. Comparisons with the honeybee and other insects allowed the identification of potential candidates for gene loss, pseudogene evolution, and possible incomplete annotation in the honeybee genome. Further, given the focus of much basic research and the perceived threat of disease to natural and commercial populations, the immune system of bumblebees is a particularly relevant component. Although the library is derived from unchallenged bees, we still uncover transcription of a number of immune genes spanning the principally described insect immune pathways. Additionally, the EST library provides a resource for the discovery of genetic markers that can be used in population level studies. Indeed, initial screens identified 589 simple sequence repeats and 854 potential single nucleotide polymorphisms.

Conclusion: The resource that these $B$. terrestris ESTs represent is valuable for ongoing work. The ESTs provide direct evidence of transcriptionally active regions, but they will also facilitate further functional genomics, gene discovery and future genome annotation. These are important aspects in obtaining a greater understanding of this key pollinator species.
\end{abstract}

\section{Background}

Social insects, especially those belonging to the order Hymenoptera, have been an extremely successful group. They take on a wide diversity of roles around the world, and while some are considered as pests, others are seen as vital for ecosystem functioning and even commercial prosperity. Bumblebees (the genus Bombus) fall into the latter beneficial groups, being important members of natural pollinator assemblages and providing commercial pollination services [1].

\footnotetext{
* Correspondence: ben.sadd@env.ethz.ch
'Institute of Integrative Biology (IBZ), ETH Zürich, Universitätsstrasse 16, 8092

* Correspondence: ben.sadd@env.ethz.ch
'Institute of Integrative Biology (IBZ), ETH Zürich, Universitätsstrasse 16, 8092 Zürich, Switzerland
}

(c) 2010 Sadd et al; licensee BioMed Central Ltd. This is an Open Access article distributed under the terms of the Creative Commons Attribution License (http://creativecommons.org/licenses/by/2.0), which permits unrestricted use, distribution, and reproduction in any medium, provided the original work is properly cited. study due to their importance, behaviour, social life, and a number of other fascinating traits. To name but a few, bumblebees have been utilised in research on social evolution $[2,3]$ and organisation $[4,5]$, development $[6,7]$, plant-pollinator interactions [8-10], learning [11,12], invasion biology [13], host-parasite ecology [14-17], ecological immunology [18-20], and community ecology $[21,22]$. While not officially domesticated, bumblebees still present an important agricultural resource with a significant economic load [23,24]. Bumblebees in both Europe (mainly B. terrestris) and North America (mainly B. impatiens) are bred commercially by the hundred thousands for this reason, with the colonies being used 
in the pollination of a variety of standard glasshouse crops [23]. Many have considered their economic importance to be on the rise given the recent declines in honeybee numbers [25]. Bumblebees not only provide valuable pollination services in the agricultural sector, but the pollination they carry out is an integral part of many natural ecosystems [26-28]. The importance of bumblebees comes sharply into focus when we consider the threat that pollinators currently face worldwide. This pollinator crisis has been epitomised by the recent honeybee colony collapse disorder [25], but the bumblebee has also suffered population declines in many areas over a number of decades [29-31].

Given their importance, a good knowledge of bumblebees from the genomic level up is of great interest. An improved set of genomic resources for bumblebees would facilitate further studies in the bumblebee, and comparisons with other bees such as the honeybee, Apis mellifera, where the genome is already sequenced [32]. There is currently a limited set of resources available for genetic investigation, and at the time of writing, for B. terrestris, there were $61 \mathrm{cDNA}$ sequences and 288 nucleotide sequences (172 of which represent microsatellite markers) in NCBI Genbank (http://www.ncbi.nlm.nih.gov, November 2009). Further tools available include a linkage map for B. terrestris [33] and a BAC library [34].

In order to greatly expand the genomic resources available for $B$. terrestris we constructed a normalised expressed sequence tag (EST) library from thorax and abdomen tissue of workers. ESTs are short, unedited, randomly selected, single pass reads from cDNA libraries [35]. They provide evidence of transcriptionally active regions in an organism and are an excellent resource for upstream work including gene discovery, functional genomics and marker discovery $[35,36]$. Standard cDNA libraries can have problems with redundancy, and rare transcripts are often under-represented. Therefore, we employed normalisation in order to increase the number of unique transcripts discovered [37].

Here we describe a collection of 29,428 ESTs, which represent a valuable resource for further ecological and evolutionary studies in Bombus spp. and for comparative studies with other Hymenoptera and insects. Sequences have been deposited in the GenBank, EMBL, and DDBJ nucleotide sequence databases under the Accession numbers FN611035 through to FN640462. For further material, including consensus sequences of assembled contigs, please contact the authors.

\section{Results and Discussion}

\section{Sequence assembly and features}

A total of 29,428 quality controlled EST sequences were assembled using TGI clustering tools (TGICL). The assembly generated 4,682 contigs and 8,651 singletons. The average number of sequences per contig was 4.43 with the maximum being 35 . Contigs averaged 960 bases in length with the longest contig being 3,542 bases. In total 13,333 unique sequences were produced. A table showing all EST and contig statistics can be found as an additional file (Additional file 1).

\section{Sequence annotation}

The 13,333 unique sequences were used in a BLASTX search against the non-redundant (nr) database in Genbank (version dated 19th October 2009). A total of 7,844 (58.8\%) ESTs had significant "hits" (cutoff $=1 \mathrm{e}-$ 05) (Table 1 and Additional File 2). Of these, the majority (7,260 sequences) had "best-hits" to known sequences within the Hymenoptera, with a large proportion (6,296 sequences) of these belonging to Apis spp A total of 5,489 sequences (41.2\%) had no significant similarity to any sequences contained in the $\mathrm{nr}$ database (Additional file 3). It is possible that many of these sequences without "hits" are cDNAs of known proteins, but consist mainly of untranslated regions (UTRs), therefore reducing the likelihood that matches are found through BLASTX. However, we cannot discount the possibility that some of the proteins are novel.

\section{Gene ontology (GO) terms}

The assembled B. terrestris ESTs were characterised for gene ontology terms in molecular function, biological process and cellular component by comparison against annotated proteins in the SwissProt and Trembl databases (UniProt Knowledgebase Release 15.9) using annot8r [38]. In order to increase the accuracy of any annotations, only GO terms that had been allocated by manual curation, and not those electronically inferred, were used. The distribution of the terms in each of the

$\begin{aligned} & \text { Table } \mathbf{1} \text { Taxonomic distribution of the “best-hits" for the } \\
& \mathbf{1 3 , 3 3 3} \text { post-assembly Bombus terrestris EST sequences } \\
& \text { (BLASTX, cutoff = 1e-05) }\end{aligned}$
\begin{tabular}{ll}
\hline Taxonomic classification & Number of assembled ESTs \\
\hline Apis spp. & 6,296 \\
Nasonia vitripennis & 886 \\
Existing Bombus terrestris sequences & 8 \\
Other Bombus spp. & 49 \\
Other Hymenoptera & 21 \\
Diptera & 179 \\
Coleoptera & 110 \\
Other Insecta & 146 \\
Other & 149 \\
No hits & 5,489
\end{tabular}

Relevant groupings have been performed, but original counts on an individual taxon identifier level can be found as an additional file (Additional file 2). 
GO Slim overview categories can be seen in Table 2, and the full GO assignments can be found as an additional file (Additional file 4). Further, the same process was completed for predicted proteins in the A. mellifera and Tribolium castaneum genomes [32,39]. This allowed for a comparison to be made with the distribution of terms that we obtained for the $B$. terrestris unique sequences in this study. General patterns of distribution were similar between organisms, with limited exceptions where particular GO slim categories were over- or under-represented in the $B$. terrestris unique sequences (Table 2).

Table 2 Comparison of the percentage distribution of Bombus terrestris Gene Ontology terms (overview GO slim terms) based on the reported EST library and the distribution of Gene Ontology terms for all proteins predicted in each of the Apis mellifera and Tribolium castaneum genomes

\begin{tabular}{|c|c|c|c|}
\hline GO slim term* & B. terrestris & A. mellifera & T. castaneum \\
\hline \multicolumn{4}{|l|}{ Molecular function } \\
\hline motor activity & $0.4 \%(14)$ & $0.9 \%$ & $0.8 \%$ \\
\hline ligase activity & $2.0 \%(64)$ & $2.1 \%$ & $2.0 \%$ \\
\hline translation regulator activity & $<0.1 \%(2)$ & $<0.1 \%$ & $<0.1 \%$ \\
\hline signal transducer activity & $1.6 \%(52)$ & $2.7 \%$ & $3.0 \%+$ \\
\hline catalytic activity & $11.8 \%(381)$ & $10.4 \%$ & $11.0 \%$ \\
\hline binding & $57.0 \%(1842)$ & $57.8 \%$ & $55.8 \%$ \\
\hline transferase activity & $9.8 \%(318)$ & $8.6 \%$ & $8.6 \%$ \\
\hline transporter activity & $4.0 \%(131)$ & $5.6 \%$ & $6.1 \%+$ \\
\hline transcription regulator activity & $2.5 \%(81)$ & $3.3 \%$ & $3.1 \%$ \\
\hline antioxidant activity & $0.1 \%(3)$ & $0.1 \%$ & $0.1 \%$ \\
\hline lyase activity & $0.7 \%(23)$ & $0.7 \%$ & $0.8 \%$ \\
\hline oxidoreductase activity & $5.2 \%(169)$ & $3.7 \%$ & $4.8 \%$ \\
\hline isomerase activity & $0.5 \%(17)$ & $0.3 \%$ & $0.3 \%$ \\
\hline enzyme regulator activity & $2.3 \%(73)$ & $2.3 \%$ & $2.0 \%$ \\
\hline structural molecule activity & $2.1 \%(67)$ & $1.3 \%$ & $1.6 \%$ \\
\hline \multicolumn{4}{|l|}{ Biological process } \\
\hline response to stimulus & $7.0 \%(274)$ & $5.9 \%$ & $6.8 \%$ \\
\hline cellular amino acid and derivative metabolic process & $1.7 \%(66)$ & $1.5 \%$ & $1.7 \%$ \\
\hline behavior & $2.8 \%(111)$ & $2.6 \%$ & $2.7 \%$ \\
\hline metabolic process & $18.4 \%(718)$ & $14.9 \%+$ & $16.2 \%$ \\
\hline cell differentiation & $5.6 \%(220)$ & $7.3 \%$ & $7.1 \%$ \\
\hline cell communication & $6.1 \%(240)$ & $8.1 \%+$ & $8.3 \%+$ \\
\hline nucleobase, nucleoside, nucleotide and nucleic acid metabolic process & $10.2 \%(400)$ & $6.9 \%+$ & $6.5 \%+$ \\
\hline extracellular structure organization & $0.3 \%(10)$ & $0.3 \%$ & $0.2 \%$ \\
\hline multicellular organismal development & $10.1 \%(394)$ & $12.0 \%$ & $11.8 \%$ \\
\hline cellular process & $11.9 \%(467)$ & $12.0 \%$ & $11.3 \%$ \\
\hline membrane fusion & $0.2 \%(8)$ & $0.2 \%$ & $0.2 \%$ \\
\hline transport & $8.7 \%(342)$ & $9.5 \%$ & $9.7 \%$ \\
\hline regulation of biological process & $14.6 \%(573)$ & $16.0 \%$ & $14.9 \%$ \\
\hline cell death & $1.3 \%(52)$ & $1.2 \%$ & $1.4 \%$ \\
\hline cell motion & $0.9 \%(37)$ & $1.4 \%$ & $1.2 \%$ \\
\hline \multicolumn{4}{|l|}{ Cellular component } \\
\hline membrane & $18.3 \%(472)$ & $22.6 \%+$ & $23.3 \%+$ \\
\hline cell & $6.6 \%(171)$ & $7.6 \%$ & $8.0 \%$ \\
\hline intracellular & $70.8 \%(1830)$ & $66.0 \%+$ & $63.6 \%+$ \\
\hline extracellular & $4.3 \%(111)$ & $3.7 \%$ & $5.1 \%$ \\
\hline
\end{tabular}

Actual counts appear in brackets after the percentage. Major categories of molecular function, biological process and cellular component are treated independently.

* Only manually curated terms were included to improve accuracy

† Also in bold. Statistically significant difference in the representation of the GO slim term between Bombus and the organism referred to in the column ( $p<0.0014$, Bonferroni-corrected Fisher's exact tests) 


\section{Comparisons to the honeybee and other insects}

The GC content of the $B$. terrestris unique sequences was calculated to be 36 percent (omitting ambiguous bases). Gene regions in the A. mellifera genome have a GC content of 29 percent, while in D. melanogaster and A. gambiae it is 44 and 47 percent, respectively [32]. The GC percentage based on these $B$. terrestris ESTs suggests that lower GC content of gene regions is not restricted to Apis, and may be a wider phenomenon. However, calculations of GC-content in Nasonia vitripennis mRNA (version dated 9th July 2007) at 46 percent hint that it is not Hymenopteran wide and further species would need to be studied to determine the exact range of this low GC-content.

We compared the $B$. terrestris unique sequences to proteins from genomes of each of four other insects, $A$. mellifera, $N$. vitripennis, T. castaneum and D. melanogaster (Figure 1, BLASTX, cutoff = 1e-05) (Additional file 5). The hierarchy of "hits" to each of these four insects reflects the evolutionary relationship between them and the bumblebee $[32,40,41]$, with the closest related of the four, A. mellifera, giving 15.9 percent more "hits" than the most distantly realted, $D$. melanogaster. Despite this, 212 unique sequences from this EST project have significant "hits" to all of these insects except for A. mellifera. Potential explanations for this discrepancy include an incomplete annotation of genes in the A. mellifera genome or loss of genes or their protein coding capacity following the split from Bombus in the lineage leading to Apis (see below).

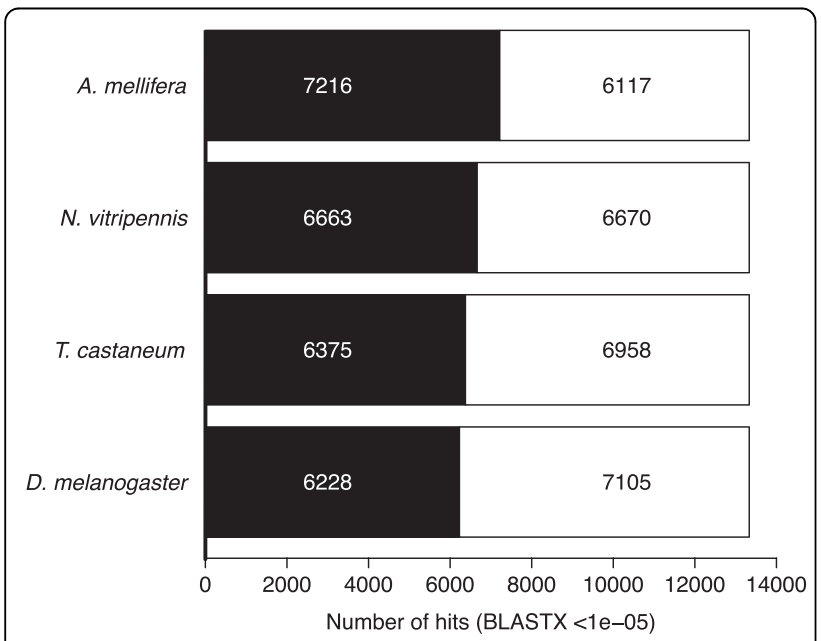

Figure 1 The number of unique Bombus terrestris sequences uncovered in this project with significant "hits" (BLASTX, cutoff $=1 \mathrm{e}-05$ ) to proteins predicted in each of the Apis mellifera, Nasonia vitripennis, Tribolium castaneum and Drosophila melanogaster genomes (black) and those with no "hits" to the predicted proteins of these organisms (white). Numbers inside bars represent the actual number of unique sequences.
The 7,216 "hits" of unique sequences to A. mellifera proteins were to 4,480 unique Apis proteins. This level of redundancy suggests that 62 percent of the assembled sequences are actually derived from unique genes, yielding an estimation of 8,278 unique genes represented by this EST library. In addition to a comparison with the predicted proteins in the $A$. mellifera genome, the unique sequences derived from the $B$. terrestris EST library were also compared to the entire $A$. mellifera genome sequence (BLASTN, cutoff $=1 \mathrm{e}-08$ ) [32]. A total of 10,024 unique sequences had "hits" to the genome, 6,749 of which already had "hits" to predicted $A$. mellifera proteins, but 3,275 which did not. These unique sequences with "hits" to the genome were distributed on the Apis linkage groups as follows, with the figure in brackets being the percent distribution of actual Apis protein coding sequences: 9.98 (9.57) percent on LG1, 5.89 (5.12) on LG2, 4.94 (4.61) on LG3, 4.94 (4.52) on LG4, 6.74 (5.77) on LG5, 3.73 (3.55) on LG6, 3.5 (3.56) on LG7, 5.25 (4.79) on LG8, 3.79 (3.76) on LG9, 5.04 (4.44) on LG10, 6.24 (5.97) on LG11, 3.20 (2.80) on LG12, 3.25 (3.03) on LG13, 4.03 (4.13) on LG14, 4.35 (4.61) on LG 15, 2.77 (2.30) on LG16, 0.02 (0.14) on the Mitochondria and 22.38 (27.34) on unassembled regions.

Particularly interesting is the apparent discrepancy between the number of $B$. terrestris unique sequences with matches to $A$. mellifera proteins and the number with matches to the $A$. mellifera genome. In fact, of the 212 unique sequences that had "hits" to all the other insect proteins tested but not Apis, 196 have "hits" to the Apis genome. There are a variety of possible reasons for a unique sequence having a "hit" to the Apis genome but not to predicted proteins from the same organism. The most obvious, is that the sequences in question are related to known Apis proteins, but they mainly contain untranslated regions (UTRs) and therefore do not produce significant "hits" in BLASTX alignments. When comparing the locations of the start of "hits" to the genome and the positions of predicted Apis protein coding sequences, 389 are either inside a protein coding sequence or span the boundary of one, suggesting that they comprise mostly of UTRs. Furthermore, 1,064 of the "hits" were within the range of possible Apis UTRs (maximum Apis UTR $=4,375$ bases, obtained from the UCSC genome browser [42]). However, this still left 1,253 sequences, including 76 of the unique sequences that had "hits" to proteins from all the other insects tested but not Apis. These 1,253 sequences were further analysed for the presence of ORFs using ORF Finder http://www.ncbi.nlm.nih.gov/gorf. The median ORF size was 225 nucleotides (range 102-1,464) suggesting that many of the unique sequences have the potential to encode proteins. While some of these "hits" to the Apis 
genome may be spurious, it is possible that they are the result of either incomplete gene annotation in the $A$. mellifera genome or evolution of pseudogenes in the Apis lineage. In an attempt to gain a further insight into these possibilities, from the 20 unique sequences with the longest predicted reading frames we inspected more closely the sequences that had a highly significant "hit" to another insect protein (BLASTX, <1e-20) (Table 3). By "blasting" these proteins against the Apis genome (TBLASTN), we attempted to find reasons that these proteins are not in the set of predicted Apis proteins. All six of the proteins had "best-hits" in the same location of the genome as the unique sequences had, and visual inspection of alignments revealed premature stop codons were coded for in the Apis sequences in four out of the six cases.

\section{Immune genes and pathways}

While the bumblebee workers used to produce this EST library were not immune challenged, the vast amount of work on immunity and host-parasite interactions in these insects [19,43-45] makes this category of genes nonetheless worthy of further interest. Furthermore, parasites have been implicated in declines of natural and commercial pollinators, such as bumblebees, worldwide $[25,46,47]$, and thus knowledge of genes and pathways involved in immunity and parasite defence is highly important.

Based on the annotations of the unique sequences, and in comparison with proposed A. mellifera immune genes [48], we find that 134 unique sequences have "best hits" to proteins from genes characterised as immune related (Additional file 6). These 107 unique sequences in Bombus terrestris match to 67 Apis genes (a similar level of redundancy to that shown with all $A$. mellifera protein "hits"). In addition, based on the classification by Sackton et al. [49], 101 unique sequences have "best-hits" in D. melanogaster to proteins of 58 immune genes. However, a core set of only 32 unique sequences had both immune classified "hits" in D. melanogaster and A. mellifera, with "hits" to 24 and 25 unique genes, respectively. This could be due differential annotation of immune genes in the two organisms, or different divergence from $B$. terrestris among different genes.

The potential immune gene transcripts found span the major described pathways in the invertebrate immune system (Figure 2), and based on these certain components, we can begin to build a picture of immune defence in bumblebees. We find that there is evidence for the existence and transcription of integral genes in each of the described pathways.

\section{Potential EST-derived genetic markers}

Traditional methods of isolating and characterising genetic markers can be expensive and time-consuming. However, ESTs can be a valuable resource for the initial identification of genetic markers that can subsequently be used in studies of molecular ecology [36]. Simple sequence repeats (SSRs) and single nucleotide polymorphisms (SNPs) allow population level studies both within the target species and related species.

Using MISA [50], a number of SSRs were identified in the unique sequences. We restricted our search for SSRs to di-, tri-, tetra-, penta-, and hexa- nucleotide motifs that were repeated at least six times for di-nucleotide and five times for all other motifs. We found a total of 589 markers found in 507 unique sequences. 397 di- $($ mean repeat length $=7.65$, range $=6-17), 154$ tri(mean repeat length $=5.79$, range $=5-17), 26$ tetra(mean repeat length $=6$, range $=5-11), 6$ penta- $($ mean repeat length $=5.83$, range $=5-7)$, and 6 hexa- (mean repeat length $=6.33$, range $=5-9$ ) nucleotide repeats were found. 61 of the SSRs were found in compound formation with another (maximum of 100 nucleotides separation). While polymorphism remains to be verified for these markers with appropriate laboratory screens, previous levels of polymorphism in EST derived SSRs

Table 3 Unique sequences from the 20 longest ORFs that have "hits" to other insect proteins and also "hits" away from protein coding regions in the Apis genome, but are not part of the Apis protein set

\begin{tabular}{|c|c|c|c|c|}
\hline $\begin{array}{l}\text { Project Identification } \\
\text { (bom001no-) }\end{array}$ & Organism "best-hit" & Protein & Description & $\begin{array}{l}\text { Potential reason } \\
\text { for absence from } \\
\text { A. mellifera protein set }\end{array}$ \\
\hline CL2097Contig1 & N. vitripennis & XP_001604128.1 & similar to F-box protein 28 & Stop codon \\
\hline CL2223Contig1 & N. vitripennis & NP_001123269.1 & nucleoporin $\mathrm{Ndc1}$ & Unknown \\
\hline CL2537Contig1 & N. vitripennis & XP_001606900.1 & similar to p53-like protein & Stop codon \\
\hline CL2610Contig1 & N. vitripennis & XP_001600663.1 & similar to ENSANGP00000017887 & Unknown \\
\hline CL2809Contig1 & N. vitripennis & XP_001604974.1 & similar to GA18228-PA & Stop codon \\
\hline P0115M15_F & P. humanus corporis & XP_002432715.1 & nuclear pore complex protein nup98 & Stop codon \\
\hline
\end{tabular}

Unique sequences, from the sequences with the 20 longest ORFs that have "hits" to the Apis genome at least 4'375 bases away from predicted Apis protein coding regions, with highly significant "best-hits" (BLASTX, 1e-20) to an insect protein. These "best-hit" insect proteins were "blasted" against the Apis genome (TBLASTN) and the resulting alignments scanned for potential elements hindering protein production. 


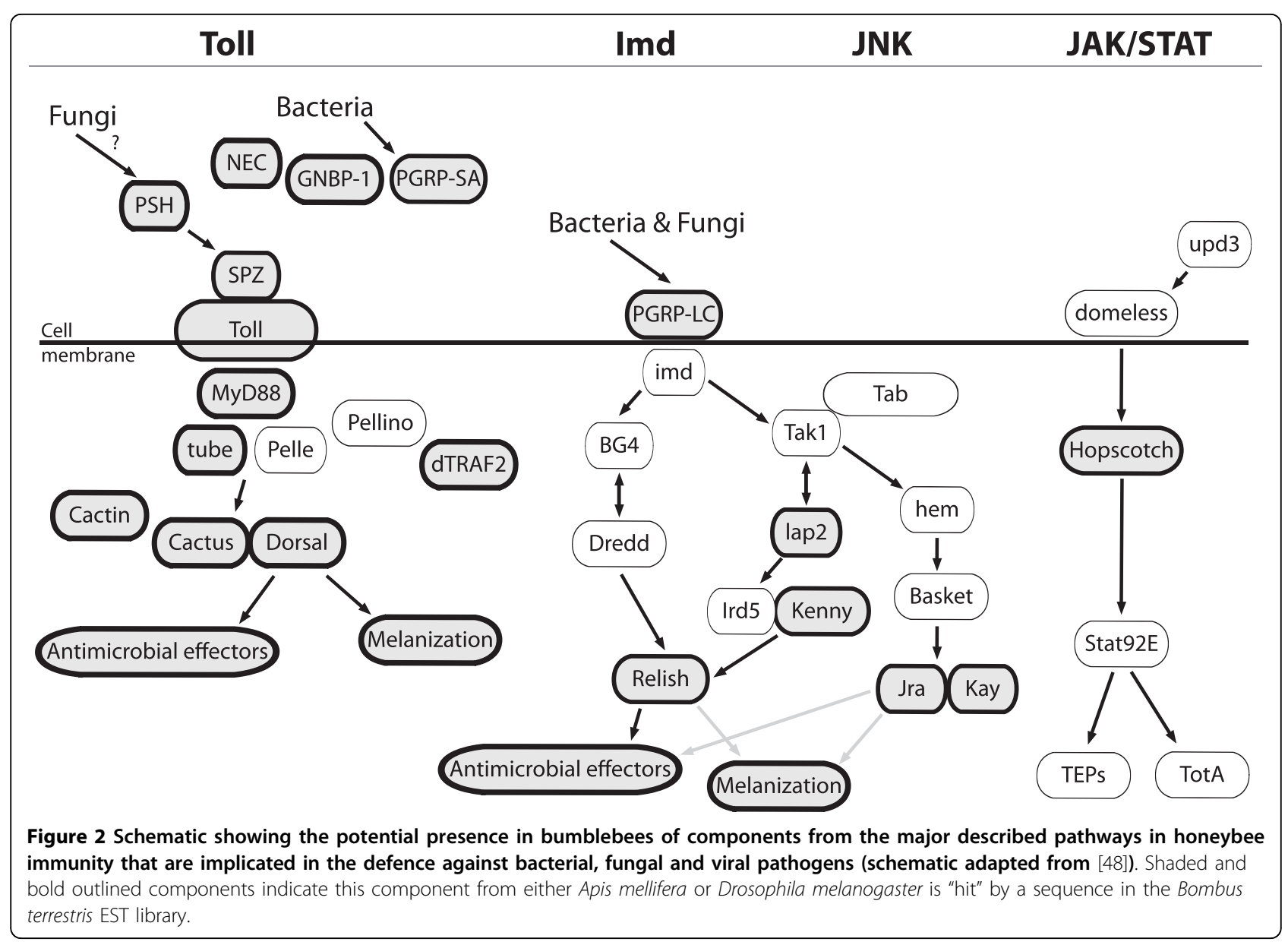

suggest that a large number of these markers will be variable [51].

These identified potential SSRs together with existing markers from B. terrestris [52-54] make up a significant resource for molecular ecology studies. While EST derived SSRs tend to show a lower level of polymorphism than those identified from genomic libraries, they can more often be used across related species $[36,51,55]$. Given the pervasive importance of species of the genus Bombus in temperate pollinator communities, this is a highly advantageous feature. In addition to SSRs, contigs built from a number of EST reads can be useful sources of potential SNPs. While the normalization procedure used in this work reduces such redundancy and thus the likelihood of finding SNPs, 1,973 contigs contain at least four sequences, a minimum for reliable identification of SNPs [56]. These contigs cover 2,124,958 bases. Using SNPidentifier [57] with minimum minor allele frequency of 0.1 and a minimum occurrence of 2 , we were able to find 854 potential SNPs occuring in 573 contigs. These potential SNPs provide an additional set of genetic markers for subsequent population studies.

\section{Conclusions}

The resource provided by the presented EST library will serve researchers in a diverse range of biological fields. The detected markers will facilitate further population level studies, and the sequences themselves can be used in investigations of particular genes, and also in functional genomic approaches. Further, as demonstrated here, the sequences allow genomic comparisons with the honeybee A. mellifera and other insects, informing on such things as potential gene loss or pseudogene evolution. This EST library will also be a practical resource in the assembly and annotation of the impending B. terrestris genome http://www.hgsc.bcm.tmc.edu. These elements, coupled with the major commercial and ecosystem functions of $B$. terrestris and related hymenoptera, mean that this EST library is a useful resource for ongoing research.

\section{Methods}

\section{Animals}

Colonies were set-up with field caught queens collected from Northern Switzerland in the spring of 2007 
(Aesch, Basel), and were kept under red-light illumination at $27 \pm 1^{\circ} \mathrm{C}$ with sugar water (Apilnvert ${ }^{\circ}$ ) and pollen provided ad libitum. Worker bees used for the EST library originated from four colonies (two workers each) that had been confirmed as Bombus terrestris by visual checks and based on a species-specific length polymorphism marker in the region between mitochondrial $\mathrm{CO} 1$ and $\mathrm{CO} 2$ genes. The colonies were also confirmed to be free of common parasites by microscopic investigation of faeces. Worker bees were age controlled, and one bee from each colony was 7 and one bee 14 days post adult eclosion at the time of sacrifice. Bees were sacrificed by snap freezing in liquid nitrogen, and were subsequently stored at $-80^{\circ} \mathrm{C}$. Tissue from both the thorax and abdomen was used as material for the EST library.

\section{RNA isolation and CDNA library construction}

Total RNA was extracted with Solution D [58]. cDNA was synthesized using the SMART approach (Mint-Universal cDNA synthesis kit, Evrogen, Russia), subsequently normalized using duplex-specific nuclease (Trimmer kit, Evrogen, Russia) according to manufacturer's instructions, and directionally cloned into pAL32 plasmid vector (Evrogen, Russia). Plasmids were transferred via electroporation into E. coli DH10B (Invitrogen, U.S.A.).

\section{EST sequencing, quality control and assembly}

Plasmids from the normalized libraries were 5 ' end sequenced using the pALforward primer (5'CTCGGGAAGCGCGCCATT-3') and Big Dye Terminator chemistry (ABI). Collected reads from 3730XL capillary sequencers $(\mathrm{ABI})$ were base-called using Phred http://www.phrap.org and subsequently quality and vector clipped using Lucy http://compbio.dfci.harvard.edu/ tgi/software with standard parameters. For clustering and assembly, the TGI tools developed at TIGR http:// compbio.dfci.harvard.edu/tgi/software were used.

\section{Annotation and comparisons}

Where explicit packages and scripts are not mentioned, results were obtained by a mixture of custom Perl scripts implementing BioPerl modules [59] and custom $\mathrm{R}$ functions in R2.9.2 for Mac [60].

Once clusters were created, the resulting unique sequences were used for various comparisons. BLASTX searches with a cut-off of 1e-05 were run against each of the nr database in Genbank (version dated 19th October 2009), and databases comprising of all predicted proteins in the genomes of A. mellifera, N. vitripennis, $D$. melanogaster and $T$. castaneum.

Redundancy in the unique sequences was determined by comparison with $A$. mellifera. This was calculated as the number of unique $A$. mellifera proteins that were "best-hits" in the BLASTX search divided by the number of unique $B$. terrestris sequences with hits. GC-content was calculated using a custom Perl script that amalgamated all unique sequences and calculated the proportion of $\mathrm{G}$ and $\mathrm{C}$ bases in all unambiguous bases.

Annotation of GO terms [61] to the current B. terrestris EST derived unique sequences were carried out using the Perl based annot8r [38]. A database containing SwissProt and Trembl (Uniprot Knowledgebase Release 15.9) with manually curated GO terms was used. Terms that had been derived electronic annotation were omitted to improve reliability of the annotations. BLASTP against this database was carried out with a cut-off of 1e-05. In addition, predicted proteins from the entire genomes of $A$. mellifera [32] and T. castaneum [39] were compared with the same database for comparisons of the distribution of GO terms. For statistical comparisons, GO terms were matched to higher-level GO Slim categories. Pairwise comparisons between $B$. terrestris and both $A$. mellifera and $T$. castaneum were then carried out for the distribution of sequences in each GO Slim category using Fisher's exact tests in R2.92 for Mac [60].

The EST unique sequences were compared with the A. mellifera genome [32] using BLASTN, cut-off 1e-08. A custom Perl script was then used to extract positional information of all the "best-hits". Linkage group and position on the linkage group was then compared with the position of predicted protein coding regions within the genome to obtain a relative position of the "hits" (inside a protein-coding region; spanning a protein-coding region boundary; outside a protein coding region). For those outside a predicted protein-coding region, the distance to the closest was calculated. For those B. terrestris sequences with hits greater than 4'375 bases (the largest $A$. mellifera UTR, obtained from the UCSC genome browser [42]) away from a protein coding region, a custom Perl script was used to interface with ORF Finder http://www.ncbi.nlm.nih.gov/gorf to obtain ORF information. Of these sequences, the 20 with the largest ORFs were investigated manually. BLASTP searches were carried out against the $\mathrm{nr}$ database, and if the "best-hit" was to another insect protein (cut-off $=1 \mathrm{e}$ 20), the "best-hit" was taken and blasted (TBLASTN) against the $A$. mellifera genome. It was confirmed that the "hit" location was the same as for the EST derived unique sequence and the Blast alignments were manually examined for potential features inhibiting protein production.

\section{SSR and SNP marker identification}

To identify potential genetic markers we used the Perl scripts MISA [50] and SNPidentifier [57] to locate 
simple sequence repeats and single nucleotide polymorphisms, respectively.

With MISA the configuration file was adjusted to identify motifs of two, three, four, five or six nucleotides, that repeat at least six times for di-nucleotide repeats and five times for all others. MISA was then run on all the post-assembly unique sequences. With SNP identifier we adjusted the minimum minor allele frequency to 0.1 and occurrence to 2 . This meant that post-assembly sequences needed to be made up of at least four ESTs to be considered. SNPidentifier was run on the alignments of the EST sequences.

Additional file 1: Assembly statistics of the Bombus terrestris ESTs.

Click here for file

[http://www.biomedcentral.com/content/supplementary/1471-2164-11110-S1.PDF ]

Additional file 2: Counts of the "best-hits" at the individual taxonomic identifier level for the 13,333 post-assembly Bombus terrestris EST sequences (BLASTX, cutoff $=1 \mathrm{e}-05$ ).

Click here for file

[http://www.biomedcentral.com/content/supplementary/1471-2164-11110-S2.XLS ]

Additional file 3: Presence and identity of "best-hits" for the 13,333 unique Bombus terrestris sequences in the non-redundant (nr) database of Genbank (version dated 19th October 2009) (BLASTX cutoff $=1 \mathrm{e}-05$ ).

Click here for file

[http://www.biomedcentral.com/content/supplementary/1471-2164-11110-S3.XLS ]

Additional file 4: Gene Ontology assignments for the unique Bombus terrestris sequences.

Click here for file

[http://www.biomedcentral.com/content/supplementary/1471-2164-11110-S4.XLS]

Additional file 5: Presence and identity of "best-hits" in paired BLASTX comparisons (cutoff $=1 \mathrm{e}-05$ ) of the unique Bombus terrestris sequences against proteins from each of Apis mellifera, Nasonia vitripennis, Tribolium castaneum and Drosophila melanogaster.

Click here for file

[http://www.biomedcentral.com/content/supplementary/1471-2164-11110-S5.XLS]

Additional file 6: Bombus terrestris unique sequences with "hits" to proteins from genes characterised as immune related in Apis mellifera and Drosophila melanogaster.

Click here for file

[http://www.biomedcentral.com/content/supplementary/1471-2164-11110-S6.XLS]

\section{Acknowledgements}

We acknowledge Monique Roenick and Janina Thiel (Berlin) for technical assistance, and Alfred Beck (Berlin) for computational support. This study was supported by the Max Planck Society, the Swiss National Science

Foundation, CCES (GEDIHAP) and the Genetic Diversity Centre of ETH Zurich.

\section{Author details}

'Institute of Integrative Biology (IBZ), ETH Zürich, Universitätsstrasse 16, 8092 Zürich, Switzerland. ${ }^{2}$ Max Planck Institute for Molecular Genetics, Ihnestrasse 73, 14195 Berlin, Germany.

\section{Authors' contributions}

BMS prepared the animals for the EST library, carried out bioinformatics analyses of the sequences. MK, SK and RR contributed to RNA isolation library construction, template preparation, sequence determination and assembly. BMS, MK, SK, RR and PSH drafted the manuscript. PSH was the initiator of this project. All authors read and approved the final manuscript.

Received: 30 November 2009

Accepted: 15 February 2010 Published: 15 February 2010

\section{References}

1. Goulson D: Bumblebees - their behaviour and ecology. New York, USA: Oxford University Press 2003.

2. Pamilo P: Evolution of Colony Characteristics in Social Insects .1. Sex Allocation. Am Nat 1991, 137(1):83-107.

3. Page RE, Metcalf RA: Multiple Mating, Sperm Utilization, and Social Evolution. Am Nat 1982, 119(2):263-281.

4. Jandt JM, Dornhaus A: Spatial organization and division of labour in the bumblebee Bombus impatiens. Anim Behav 2009, 77(3):641-651.

5. Schmid-Hempel P, Schmid-Hempel R: Transmission of a pathogen in Bombus terrestris, with a note on division of labor in social insects. Behavioral Ecology and Sociobiology 1993, 33(5):319-327.

6. Yerushalmi $\mathrm{S}$, Bodenhaimer $\mathrm{S}$, Bloch $\mathrm{G}$ : Developmentally determined attenuation in circadian rhythms links chronobiology to social organization in bees. J Exp Biol 2006, 209(6):1044-1051.

7. Hartfelder K, Cnaani J, Hefetz A: Caste-specific differences in ecdysteroid titers in early larval stages of the bumblebee Bombus terrestris. J Insect Physiol 2000, 46(11):1433-1439.

8. Cooley AM, Carvallo G, Willis JH: Is floral diversification associated with pollinator divergence? Flower shape, flower colour and pollinator preference in chilean Mimulus. Annals of Botany 2008, 101(5):641-650.

9. Suzuki K, Dohzono I, Hiei K: Evolution of pollinator generalization in bumblebee-pollinated plants. Plant Species Biology 2007, 22(3):141-159.

10. Gegear RJ, Burns JG: The birds, the bees, and the virtual flowers: Can pollinator Behavior drive ecological speciation in flowering plants?. Am Nat 2007, 170(4):551-566

11. Ings TC, Raine NE, Chittka L: A population comparison of the strength and persistence of innate colour preference and learning speed in the bumblebee Bombus terrestris. Behavioral Ecology and Sociobiology 2009, 63(8):1207-1218

12. Leadbeater $E$, Chittka $L$ : The dynamics of social learning in an insect model, the bumblebee (Bombus terrestris). Behavioral Ecology and Sociobiology 2007, 61(11):1789-1796.

13. Schmid-Hempel P, Schmid-Hempel R, Brunner PC, Seeman OD, Allen GR: Invasion success of the bumblebee, Bombus terrestris, despite a drastic genetic bottleneck. Heredity 2007, 99(4):414-422.

14. Otti O, Schmid-Hempel P: Nosema bombi: A pollinator parasite with detrimental fitness effects. J Invertebr Pathol 2007 96(2):118-124

15. Baer B, Schmid-Hempel P: Bumblebee workers from different sire groups vary in susceptibility to parasite infection. Ecol Lett 2003, 6(2):106-110.

16. Durrer S, Schmid-Hempel P: Shared Use of Flowers Leads to Horizontal Pathogen Transmission. Proc R Soc Lond Ser B-Biol Sci 1994, 258(1353):299-302

17. Schmid-Hempel P, Reber Funk C: The distribution of genotypes of the trypanosome parasite, Crithidia bombi, in populations of its host, Bombus terrestris. Parasitology 2004, 129:147-158.

18. Doums C, Moret Y, Benelli E, Schmid-Hempel P: Senescence of immune defence in Bombus workers. Ecol Entomol 2002, 27(2):138-144.

19. Moret Y, Schmid-Hempel P: Survival for immunity: The price of immune system activation for bumblebee workers. Science 2000, 290(5494):1166-1168.

20. Sadd BM, Schmid-Hempel P: Facultative but persistent transgenerational immunity via the mother's eggs in bumblebees. Current Biology 2007, 17(24):R1046-R1047.

21. Hatfield RG, LeBuhn G: Patch and landscape factors shape community assemblage of bumble bees, Bombus spp. (Hymenoptera: Apidae), in montane meadows. Biological Conservation 2007, 139(1-2):150-158.

22. Williams PH, Araujo MB, Rasmont P: Can vulnerability among British bumblebee (Bombus) species be explained by niche position and breadth?. Biological Conservation 2007, 138(3-4):493-505. 
23. Velthuis HHW, van Doorn A: A century of advances in bumblebee domestication and the economic and environmental aspects of its commercialization for pollination. Apidologie 2006, 37(4):421-451.

24. Palma G, Quezada-Euan JJG, Reyes-Oregel V, Melendez V, Moo-Valle H: Production of greenhouse tomatoes (Lycopersicon esculentum) using Nannotrigona perilampoides, Bombus impatiens and mechanical vibration (Hym.: Apoidea). Journal of Applied Entomology 2008, 132(1):79-85.

25. Cox-Foster D, Vanengelsdorp D: Saving the HONEYBEE. Scientific American 2009, 300(4):40.

26. Bingham RA, Ort AR: Efficient pollination of alpine plants. Nature 1998 391(6664):238-239

27. Ono A, Dohzono I, Sugawara T: Bumblebee pollination and reproductive biology of Rhododendron semibarbatum (Ericaceae). Journal of Plant Research 2008, 121(3):319-327.

28. Forup ML, Henson KSE, Craze PG, Memmott J: The restoration of ecological interactions: plant-pollinator networks on ancient and restored heathlands. Journal of Applied Ecology 2008, 45(3):742-752.

29. Biesmeijer JC, Roberts SPM, Reemer M, Ohlemuller R, Edwards M, Peeters T, Schaffers AP, Potts SG, Kleukers R, Thomas CD, et al: Parallel declines in pollinators and insect-pollinated plants in Britain and the Netherlands. Science 2006, 313(5785):351-354.

30. Grixti JC, Wong LT, Cameron SA, Favret C: Decline of bumble bees (Bombus) in the North American Midwest. Biological Conservation 2009, 142(1):75-84.

31. Fitzpatrick U, Murray TE, Paxton RJ, Breen J, Cotton D, Santorum V, Brown MJF: Rarity and decline in bumblebees - A test of causes and correlates in the Irish fauna. Biological Conservation 2007, 136(2):185-194.

32. Honeybee Genome Sequencing Consortium: Insights into social insects from the genome of the honeybee Apis mellifera. Nature 2006, 443(7114):931-949.

33. Wilfert L, Gadau J, Schmid-Hempel P: A core linkage map of the bumblebee Bombus terrestris. Genome 2006, 49(10):1215-1226.

34. Wilfert L, Torres MM, Reber-Funk C, Schmid-Hempel R, Tomkins J, Gadau J, Schmid-Hempel P: Construction and characterization of a BAC-library for a key pollinator, the bumblebee Bombus terrestris L. Insectes Sociaux 2009, 56(1):44-48.

35. Nagaraj SH, Gasser RB, Ranganathan S: A hitchhiker's guide to expressed sequence tag (EST) analysis. Briefings in Bioinformatics 2007, 8(1):6-21.

36. Bouck A, Vision T: The molecular ecologist's guide to expressed sequence tags. Molecular Ecology 2007, 16(5):907-924.

37. Bonaldo MDF, Lennon G, Soares MB: Normalization and subtraction: Two approaches to facilitate gene discovery. Genome Research 1996, 6(9):791-806.

38. Schmid R, Blaxter M: annot8r: GO, EC and KEGG annotation of EST datasets. BMC Bioinformatics 2008, 9(1):180.

39. Tribolium Genome Sequencing Consortium: The genome of the model beetle and pest Tribolium castaneum. Nature 2008, 452(7190):949-955.

40. Savard J, Tautz D, Richards S, Weinstock GM, Gibbs RA, Werren JH, Tettelin H, Lercher MJ: Phylogenomic analysis reveals bees and wasps (Hymenoptera) at the base of the radiation of Holometabolous insects. Genome Research 2006, 16(11):1334-1338.

41. Sharkey MJ: Phylogeny and classification of Hymenoptera. Zootaxa 2007, 1668: 521-548.

42. Karolchik D, Hinrichs AS, Furey TS, Roskin KM, Sugnet CW, Haussler D, Kent WJ: The UCSC Table Browser data retrieval tool. Nucleic Acids Res 2004, 32:D493-D496.

43. Baer B, Schmid-Hempel P: Experimental variation in polyandry affects parasite loads and fitness in a bumble-bee. Nature 1999, 397(6715):151-154

44. Schmid-Hempel P: On the evolutionary ecology of host-parasite interactions: addressing the question with regard to bumblebees and their parasites. Naturwissenschaften 2001, 88(4):147-158.

45. Sadd BM, Schmid-Hempel P: Insect immunity shows specificity in protection upon secondary pathogen exposure. Current Biology 2006, 16(12):1206-1210.

46. Colla SR, Otterstatter MC, Gegear RJ, Thomson JD: Plight of the bumble bee: Pathogen spillover from commercial to wild populations. Biological Conservation 2006, 129(4):461-467.
47. Thorp RW: Species Profile: Bombus franklini. Red list of pollinator insects of North America Portland, Oregon, USA: The Xerces Society for Invertebrate ConservationShepherd M, Vaughan, DM, Black SH 2005.

48. Evans JD, Aronstein K, Chen YP, Hetru C, Imler JL, Jiang H, Kanost M, Thompson GJ, Zou Z, Hultmark D: Immune pathways and defence mechanisms in honey bees Apis mellifera. Insect Mol Biol 2006, 15(5):645-656.

49. Sackton TB, Lazzaro BP, Schlenke TA, Evans JD, Hultmark D, Clark AG: Dynamic evolution of the innate immune system in Drosophila. Nat Genet 2007, 39(12):1461-1468.

50. MISA - MIcroSAtellite identification tool. http://pgrc.ipk-gatersleben.de/ misa/.

51. Ellis JR, Burke JM: EST-SSRs as a resource for population genetic analyses. Heredity 2007, 99(2):125-132.

52. Funk CR, Schmid-Hempel $R$, Schmid-Hempel P: Microsatellite loci for Bombus spp. Molecular Ecology Notes 2006, 6(1):83-86.

53. Stolle E, Rohde M, Vautrin D, Solignac M, Schmid-Hempel P, SchmidHempel R, Moritz RFA: Novel microsatellite DNA loci for Bombus terrestris (Linnaeus, 1758). Molecular Ecology Resources 2009, 9(5):1345-1352.

54. Estoup A, Solignac M, Cornuet JM, Goudet J, Scholl A: Genetic differentiation of continental and island populations of Bombus terrestris (Hymenoptera: Apidae) in Europe. Molecular Ecology 1996, 5(1):19-31.

55. Pashley $\mathrm{CH}$, Ellis JR, McCauley DE, Burke JM: EST databases as a source for molecular markers: Lessons from Helianthus. Journal of Heredity 2006, 97(4):381-388.

56. Long $A D$, Beldade $P$, Macdonald SJ: Estimation of population heterozygosity and library construction-induced mutation rate from expressed sequence tag collections. Genetics 2007, 176(1):711-714.

57. Gorbach DM, Hu ZL, Du ZQ, Rothschild MF: SNP discovery in Litopenaeus vannamei with a new computational pipeline. Animal Genetics 2009, 40(1):106-109.

58. Chomczynski P, Sacchi N: Single-step method of RNA Isolation by acid guanidinium thiocyanate phenol chloroform extraction. Anal Biochem 1987, 162(1):156-159.

59. Stajich JE, Block D, Boulez K, Brenner SE, Chervitz SA, Dagdigian C, Fuellen G, Gilbert JGR, Korf I, Lapp H, et al: The Bioperl Toolkit: Perl Modules for the Life Sciences. Genome Research 2002, 12(10):1611-1618.

60. R: A language and environment for statistical computing. http://www.Rproject.org.

61. Gene Ontology Consortium: Gene Ontology: tool for the unification of biology. Nat Genet 2000, 25(1):25-29.

doi:10.1186/1471-2164-11-110

Cite this article as: Sadd et al:: Analysis of a normalised expressed sequence tag (EST) library from a key pollinator, the bumblebee Bombus terrestris. BMC Genomics 2010 11:110.

\section{Submit your next manuscript to BioMed Central and take full advantage of:}

- Convenient online submission

- Thorough peer review

- No space constraints or color figure charges

- Immediate publication on acceptance

- Inclusion in PubMed, CAS, Scopus and Google Scholar

- Research which is freely available for redistribution

Submit your manuscript at www.biomedcentral.com/submit
C Biomed Central 